\title{
Research on the Improved Method of Monocular Distance Measurement in Forest Fire Prevention
}

\author{
Shaoqing Tian \\ Business Administration Postdoctoral Station, Harbin University of Commerce, Harbin, China
}

\begin{abstract}
In the context of the research of fire location of forest fire, based on the existing two-dimensional data of the camera, a new method is proposed. This method improves the accuracy of the monocular distance measurement system by adding a dimension of information. Through the gray scale projection electronic image stabilization method, it achieves the automation of monocular distance measurement. Simulation experiment shows that it can better measure the distance between the camera and the target with the proposed method.
\end{abstract}

Keywords-monocular; gray projection; positioning; distance measurement

\section{INTRODUCTION}

Forest fires have always been very serious natural disasters. How to accurately and quickly locate the fire point and measure distance is a key issue when the forest fires occur. Many methods can do this in different countries, such as satellite positioning, aircraft cruising, watchtower observation (which is the main method in China) and so on [1]. Among them, the method of the watchtower observation has the advantages of low cost, timeliness and continuity, but also can not be precise location and other shortcomings. In this context, this paper examines this issue to contribute to improving the method of monocular distance measurement.

At present, there are already a large number of scholars and researchers in the distance measurement aspects. Li (2017) [2] designs a monocular distance measurement system based on Hi3516A and ODROID-C2. Hi3516A provides data acquisition function and ODROID-C2 provides data processing function, and then the results of the actual test show that the accuracy of ranging can reach $20 \mathrm{~cm}$ or less. Li (2016) [3] designs an embedded vision distance measurement system that includes a processing module with ARM Cotex-A9 i.MX6Q as a microprocessor and an acquisition module with a USB camera as a data source for human-machine collaboration, and realized the monocular vision distance measurement and location. Cheng (2016) [4] studies a monocular distance measurement algorithm for patrol line robot vision navigation for accurate and rapid positioning of obstacles on high-voltage lines, and to achieve effective traversal barrier. The test results show that the proposed method has a measurement error of less than $5 \%$ within $5000 \mathrm{~mm}$ and a measurable distance of at least $7.4 \mathrm{~m}$. Liu (2014) [5] studies an embedded vehicle distance measurement scheme based on Field-Programmable Gate Array (FPGA) to reduce the probability of occurrence of traffic rear-end accidents. Some of the work is done in these areas, such as algorithms of image acquisition, image processing, and monocular distance measurement based on small pore imaging principle, etc. Luo (2013) [6] studies the obstacle detection from the perspective of computer vision and realized the measurement of the distance of the obstacle by monocular distance measurement technology. Huang (2000) [7] studies the front obstacle identification and highway geometric model, and proposes a real-time and effective method for calculating the obstacle distance on the highway to achieve monocular distance measurement by the computer vision principle.

Based on study on monocular distance measurement, we found that there are deficiencies in the monocular distance measurement method, and proposed an improved monocular distance measurement method based on the idea of a mobile monocular system. Through the simulation experiment, the effectiveness of this idea is confirmed.

\section{RELATED THEORIES OVERVIEW}

\section{A. Monocular Distance Measurement}

The watchtower is usually single to monitor the forest situation of a region. Usually, some information of watchtower, such as the location, pitch and horizontal angle, is known, as the Figure I shows. The distance $d$ can be measure by a simple calculation as follows Formula (1):

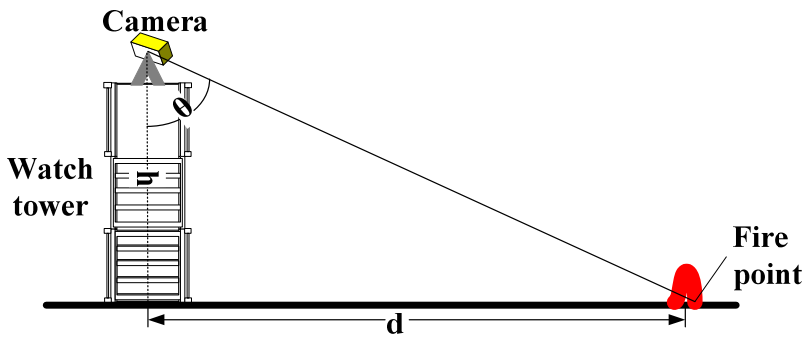

FIGURE I. MONOCULAR VISION POSITIONING METHOD

$$
d=h \sin (\theta)
$$

$\theta$ is the elevation of the camera, $\mathrm{h}$ is the distance from the camera to the ground.

\section{B. Electronic Image Stabilization Based on Gray Projection Algorithm}

EIS (Electronic Image Stabilization) [8] is a way that we can use the method of digital image processing to make images 
stable. It includes digital image acquisition through electronic equipment DV, DC, camera, digital image processing and digital image output and so on.

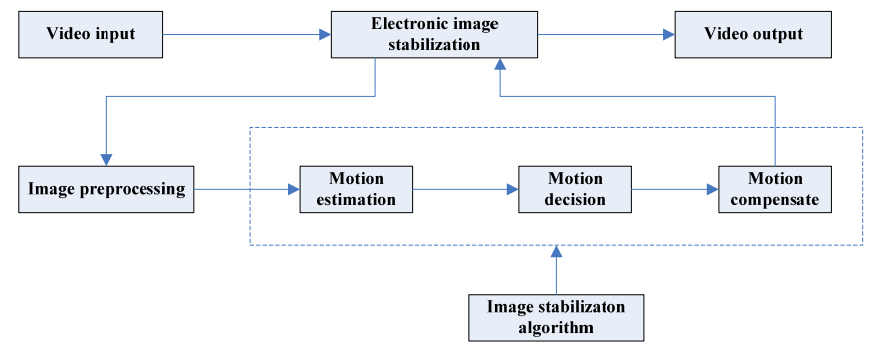

FIGURE II. FLOW CHART OF ELECTRONIC IMAGE STABILIZATION

GPA includes four steps as follows:

- Step 1: It is necessary to change the image into grayscale image.

- Step 2: It will get the row and the column projecting value through gray projection operation. Computing formula is as follows.

$$
\begin{gathered}
\operatorname{row}(i)=\sum_{j=1}^{n} \operatorname{pic}(i, j) \\
\operatorname{column}(j)=\sum_{i=1}^{m} \operatorname{pic}(i, j)
\end{gathered}
$$

$\operatorname{row}(i)$ is the row projecting value on $i$ line. column $(j)$ is the column projecting value on $j$ series. $\operatorname{pic}(i, j)$ is the gray value on the point of $(i, j)$.

- $\quad$ Step 3: The image dithering offset will be got through correlation operation. This formula is as follows.

$$
C(w)=\sum_{j=1}^{n}[\operatorname{column}(j+w-1)-\operatorname{column}(M+j)]^{2} ; 1 \leq w \leq 2 M+1
$$

$C(w)$ is the correlation operation value. $\mathrm{M}$ is the maximum extent of detection jitter in image. $W_{\min }$ is the $W$ when $C(w)$ is minimum.

Now, the offset can be computed through the following formula. The column offset is computed, so is the row offset.

$$
\text { motion }_{C}=M+1-W_{\min }
$$

- Step 4: Move the dithering image motion ${ }_{r}$ and motion ${ }_{c}$ volumes in the opposite direction, and then we can acquire the stable image.

\section{IMPROVED MONOCUlAR DistANCE MEASUREMENT METHOD}

At present, the existing watchtower monitoring equipment has two dimensions parameters of horizontal rotation (rotation angle of $360^{\circ}$ ) and longitudinal rotation (rotation angle less than $180^{\circ}$ or even $90^{\circ}$ ), as the Figure III shows.
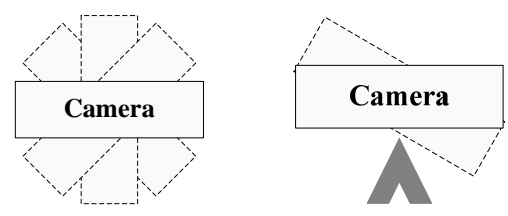

FIGURE III. HORIZONTAL ROTATION AND LONGITUDINAL ROTATION

We try to increase the accuracy of monocular distance measurement by adding a dimension on the basis of existing data, the displacement information, as the Figure IV shows.

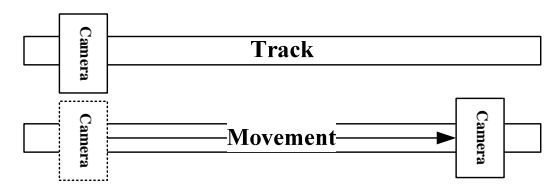

\section{FIGURE IV. MOBILE MONOCULAR MONITORING EQUIPMENT DIAGRAM}

After the system is designed, it can be used to measure the distance of fire point, which is calculated by trigonometric function in the measurement process. In order for the device to automatically acquire the corresponding data, the grayscale projection stabilization algorithm (See above 2.Electronic Image Stabilization based on Gray Projection Algorithm) can be applied to determine the horizontal rotation angle of the camera. Each frames of the image shows a horizontal displacement when the camera is moving. The gray scale projection stabilization algorithm can better calculate the displacement of the image for this situation. When the camera stops moving horizontally, rotate the camera until the offset of the image is zero, where you can get the rotation angle. The distance between the monitoring device and the target point can be calculated by the following trigonometric formula:

$$
D=x / \tan (\pi / 2-\theta) ;(0<\theta<\pi / 2)
$$

The flow chart and schematic diagram are given as shown in Figure V. 

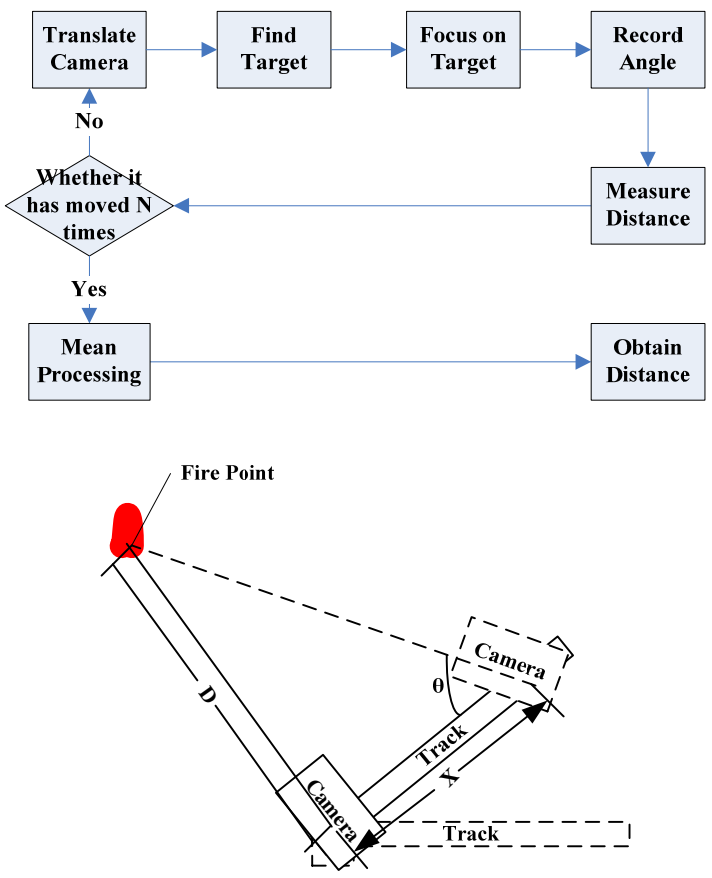

FIGURE V. FLOW CHART AND SCHEMATIC DIAGRAM FOR MEASURING DISTANCE

The specific steps are as follows seven steps:

- Step 1: Focus on the target, collect the image 1, record the angle $\theta 1$;

- Step 2: Move the device to the right, record the displacement;

- Step 3: The image is processed with a grayscale projection electronic image stabilization algorithm when the camera is rotated counterclockwise;

- Step 4: Determine if the offset (calculated by the algorithm) is zero;

- Step 5: If the offset is not zero, return to Step 3; if the offset is zero, record the angle $\theta 2$;

- Step 6: Get the angle $\theta=|\theta 1-\theta 2|$, and return $\theta$ to system;

- $\quad$ Step 7: The camera returns to its original position.

According to the above steps, the program can automatically control the rotation and movement of equipment, and can measure the distance of the target.

\section{SIMULATION EXPERIMENT}

The improved method described above is a theoretical description and has not yet been developed. Therefore, simulation experiments are carried out using conventional camera equipment (Iphone or digital camera, etc). Take a set of images as an example to illustrate the key steps of determining the rotation angle by the image stabilization, as shown in Figure VI.
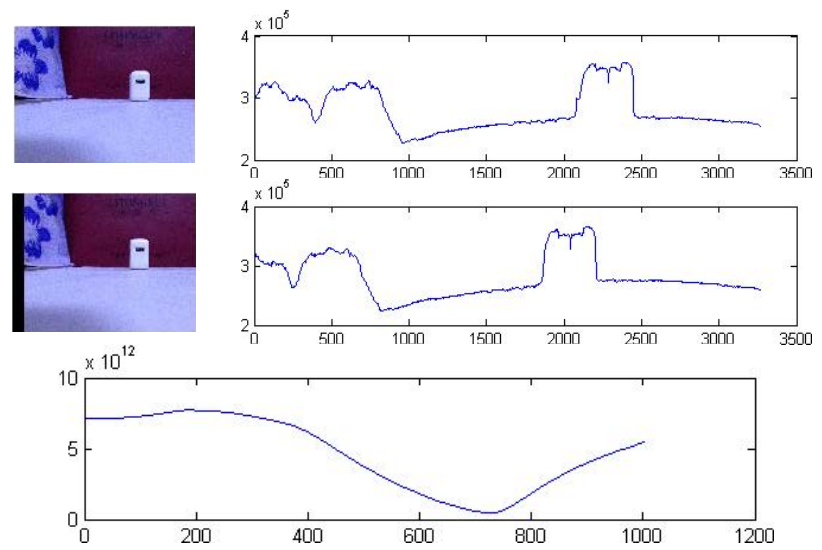

FIGURE VI. COLUMN PROJECTION CURVES AND CORRELATION CURVE ABOUT TEST IMAGES

TABLE I. EXPERIMENTAL DATA AND RESULTS

\begin{tabular}{ccccc}
\hline No. & $\begin{array}{c}\text { Camera } \\
\text { Offset(cm) }\end{array}$ & $\begin{array}{c}\text { Image } \\
\text { Offset(Pixels) }\end{array}$ & Angel $\left({ }^{\circ}\right)$ & Distance \\
\hline 1 & 1.00 & 116 & 2.27 & 25.2272 \\
2 & 2.00 & 224 & 4.57 & 25.0215 \\
3 & 3.00 & 342 & 6.84 & 25.0102 \\
4 & 4.00 & 479 & 9.10 & 24.9728 \\
5 & 5.00 & 597 & 11.29 & 25.0453 \\
6 & 6.00 & 709 & 13.53 & 24.9342 \\
7 & 7.00 & 816 & 15.67 & 24.9534 \\
8 & 8.00 & 944 & 17.75 & 24.9919 \\
\hline
\end{tabular}

Specific experimental data and description shown in Table 1, and the experimental results are given as shown in Figure VII.

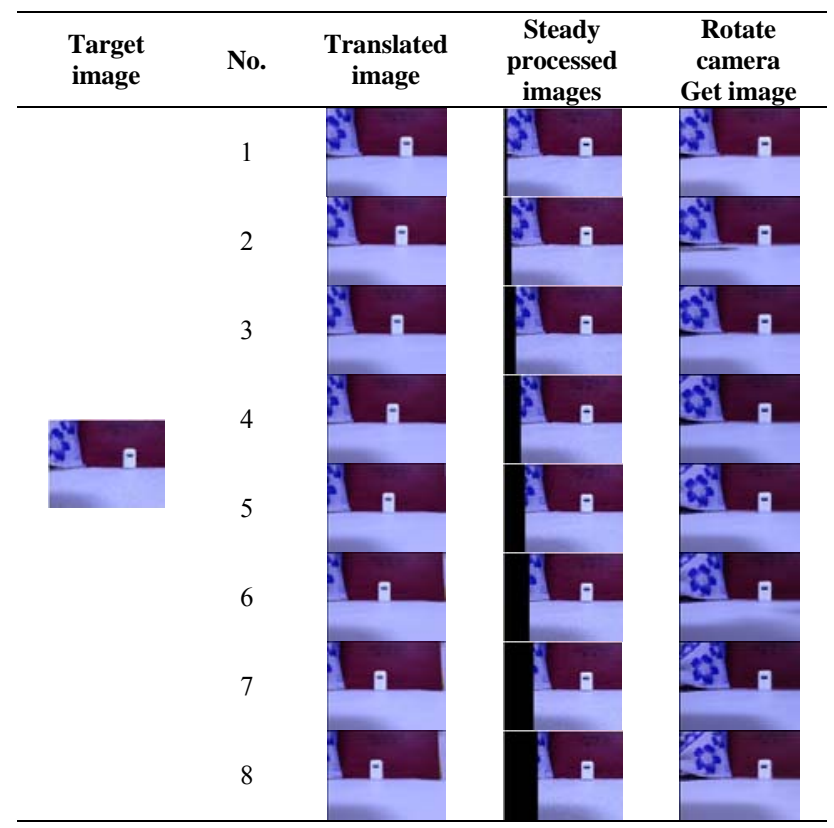

FIGURE VII. EXPERIMENTAL DATA AND RESULTS 
It may cause some error to exist because it is artificially measured, and it can reduce the corresponding error when the system mechanized.

\section{CONCLUSIONS}

How to determine the fire point as soon as possible and measure the distance is one of the key technologies to realize forest fire prevention and control automation. Monocular distance measurement is the most suitable image processing method for existing forest fire monitoring system. In this paper, the platform and method of monocular distance measurement are changed to optimize the measurement effect. Simulation experimental result shows, the proposed method can better realize the function of measuring distance automatically. Therefore, it has a certain contribution to the research of monocular distance measurement.

\section{ACKNOWLEDGMENT}

This study is supported by Harbin science and technology bureau innovation talent special funds (Youth reserve talent plan category) project. NO. 2016RAQXJ045.

\section{REFERENCES}

[1] Tian Shaoqing, Wang Nihong, Qi Hong, "A HSI seasonal adaptive method for forest monitor based on gray projection image stabilization algorithm," International Journal of Applied Mathematics and Statistics, vol. 51, pp. 81-89, 2013.

[2] Li Guoliang, Gu Minghui, Lu Jianhan, "Design and Implementation of Monocular Distance Measurement System Based on Hi3516A," Electronic Technology and Software Engineering, vol. 08, pp. 119-120, 2017.

[3] Li Xianhui, Gao Ying, Qian Gongbin, "Monocular Distance Measurement system based on Pupil Location," Intelligent Computer \& Application, vol. 02, pp. 70-73, 2016.

[4] Cheng Li, Wu Gongping, "Study on Uniform Range Finding in Visual Navigation of High-voltage Line Inspection Robot," Photonics- Laser, vol. 09, pp. 941-948, 2016.

[5] Liu Qiang, Pan Ming, Li Yongwei, "Design of Vehicle Monocular Distance Measurement System Based on FPGA," Liquid Crystal \& Display, vol. 03, pp. 422-428, 2014.

[6] Luo Shiguang, "Study on Road Obstacle Detection based on Monocular Distance Measurement Technology," Software, vol. 09, pp. 100-102 + 106, 2013.

[7] Huang Xiyue, Wang Xianzhong, Chai Yi, "Monocular Distance Measurement Algorithm based on Road Geometry Model," Journal of Chongqing University (Natural Science Edition), vol. 03, pp. 70-73, 2000 .

[8] Wang Nihong, Tian Shaoqing, Zhao Peng, "TRIZ theory is used to improve based on gray projection algorithm for electronic image stabilization," Applied Mechanics and Materials, vol. 58-60, pp. 20172022, 2011. 